\title{
Differential Metabolic Alterations and Biomarkers Between Gastric Cancer and Colorectal Cancer: A Systematic Review and Meta-Analysis
}

This article was published in the following Dove Press journal: OncoTargets and Therapy

Jingshen Tian, ', ${ }^{*}$ Weinan Xue, ${ }^{2, *}$ Huihui Yin,' Nannan Zhang,' Junde Zhou, ${ }^{3}$ Zhiping Long, (D) Chengwei $\mathrm{Wu},{ }^{3}$ Zhengzi Liang, Kun Xie, (iD)' Shuo Li, ${ }^{\prime}$ Liangliang $\mathrm{Li}$,' Zhen Wu, Volontovich Daria, (D) Yashuang Zhao, (D)' Fan Wang, (D) Maoqing Wang ${ }^{5}$

'Department of Epidemiology, School of Public Health, Harbin Medical University, Harbin,

Heilongjiang Province, People's Republic of China; ${ }^{2}$ Department of Colorectal Surgery, The Tumor Hospital of Harbin Medical University, Harbin,

Heilongjiang Province, People's Republic of China; ${ }^{3}$ Department of Colorectal Surgery, The Second Affiliated Hospital of Harbin Medical University, Harbin, Heilongjiang Province, People's Republic of China; ${ }^{4}$ Department of Colorectal Surgery, The Affiliated Hospital of Guizhou Medical University, Guiyang, Guizhou Province, People's Republic of China; ${ }^{5}$ National Key Disciplines of Nutrition and Food Hygiene, Department of Nutrition and Food Hygiene, School of Public Health, Harbin Medical University, Harbin, Heilongjiang Province, People's Republic of China

*These authors contributed equally to this work

Correspondence: Fan Wang

Department of Epidemiology, School of Public Health, Harbin Medical University, I57 Baojian Road, Nangang District.

Harbin, Heilongjiang Province, People's

Republic of China

Tel + 0086-(0)45I-87502960

$\mathrm{Fax}+0086-(0) 45 \mathrm{I}-87502885$

Email yifan.70I@I63.com

Maoqing Wang

National Key Disciplines of Nutrition and Food Hygiene, Department of Nutrition and Food Hygiene, School of Public Health, Harbin Medical University, Harbin, Heilongjiang Province, People's Republic of China

Tel + 0086-(0)45I-87502876

Fax + 0086-(0)45I-87502885

Email wang_maoqing@I26.com
Purpose: Numerous metabolomics studies have been conducted to detect the metabolic mechanisms and biomarkers related to gastric cancer and colorectal cancer. Because of the common metabolic features between gastric cancer and colorectal cancer, a differential diagnosis is difficult. Here, we performed a systematic review and meta-analysis to identify differential metabolic biomarkers between these two types of cancers.

Materials and Methods: PubMed, Embase, and ScienceDirect were searched to identify all metabolomics studies of gastric cancer and colorectal cancer published up to September 2018. Differential metabolites or altered pathways were extracted. The intersections and differences for these metabolites and pathways between gastric cancer and colorectal cancer were compared. Candidate biomarker sets for diagnosis were proposed from biofluid or feces by comparing them with tumor tissues.

Results: Totally, 24 and 65 studies were included in gastric cancer and colorectal cancer, and 223 and 472 differential metabolites were extracted, respectively. Eight pathways were reproducibly enriched in blood, tissue and urine in gastric cancer, while, 11 pathways were reproducibly enriched in blood, urine, feces and tissue in colorectal cancer. Candidate metabolic biomarker sets in blood, urine, or feces for these two cancers were proposed. We found 27 pathways (categorized into eight classifications) common to both cancers, five pathways involving 35 metabolites enriched only in gastric cancer, and eight pathways involving 54 metabolites enriched only in colorectal cancer.

Conclusion: The altered metabolic pathways showed signatures of abnormal metabolism in gastric cancer and colorectal cancer; the potential metabolic biomarkers proposed in this study have important implications for the prospective validation of gastric cancer and colorectal cancer.

Keywords: metabolomics study, colorectal cancer, gastric cancer, metabolic biomarker

\section{Introduction}

Gastric cancer (GC) and colorectal cancer (CRC) are the most common malignancies worldwide. The American Cancer Society estimates that the number of new cases of CRC in males and females in the US is 75,610 and 64,640 in 2018, ${ }^{1}$ with the number of deaths estimated at 27,390 and 23,240, respectively. This puts CRC as the third highest cancer among both men and women. Meanwhile, the estimated number of new cases of GC is 16,520 and 9,720, and those of deaths are 6,510 and 4,290 for males and females, respectively. Despite a downtrend in GC morbidity and mortality over the past decade, ${ }^{2}$ population growth and aging still have led to 
a large and rising number of new cases. It is noteworthy that the incidence of $\mathrm{GC}$ is particularly high amongst Asians and Pacific Islanders. ${ }^{3}$

Because GC and CRC are both tumors of the digestive tract, they share certain characteristics. Cancer develops slowly, often over decades, progressing from normal epithelial cells of the gastric or intestinal mucosal cells through the polyp stage to the tumor stage. Most patients are diagnosed at an advanced stage, thus missing the optimal stage for treatment. According to the TNM classification of colon cancer, the 5-year stage-specific relative survival rate decreases from $97.1 \%$ for T1/T2, N0 of Stage I to $27.1 \%$ for T4, N2 of Stage IIIC. ${ }^{4}$ Comparatively, the overall 5 -year relative survival rate for people with stomach cancer in the United States is approximately $31 \%{ }^{5}$

Clinically, endoscopy has long been considered the gold standard for a diagnosis of GC and CRC., ${ }^{6,7}$ However, its invasiveness and expense mean that it is not widely adopted by patients or for screening high-risk populations. Alternatively, blood biomarkers, such as carcinoembryonic antigen (CEA), carbohydrate antigen (CA) 19-9, and CA-125 are commonly detected but their sensitivity and specificity are unsatisfactory and are unable to differentiate between the two types of tumors. ${ }^{8}$ Therefore, there is a pressing need to identify new biomarkers for the non-invasive screening and early diagnosis of these two cancers.

Metabolomics is the endpoint of the "-omics cascade" and reflects perturbations in metabolites within all biological processes. ${ }^{9}$ Because of its high sensitivity, metabolomics offers notable advantages in identifying perturbations at an early stage of disease. Numerous small-molecule metabolites involved in various biological pathways have been identified through high-throughput analytical technology. Much effort has been made to monitor the qualitative and quantitative changes in small molecules specific to GC and $\mathrm{CRC},{ }^{10,11}$ which can help to understand the mechanisms underlying tumor formation and potentially uncover diagnostic markers. Evidence has shown that changes in metabolites are sufficiently pronounced to be detected as early indicators of diseases, and advances in global metabolomics could be used to identify individuals who may be at risk for progression from premalignant lesions to cancer. ${ }^{12}$ However, to date, hundreds of metabolic biomarkers for GC and CRC have been reported, which brought hardship on prospective validation and clinic application. Moreover, metabolomics cannot detect cancer per se like pathological sectioning, but rather detects metabolic perturbations in physiological processes. Consequently, because of the common metabolic features between $\mathrm{GC}$ and $\mathrm{CRC}$, a differential diagnosis is difficult.

Here, we systematically reviewed all metabolomics studies with a focus on the identification of metabolic biomarkers in GC and CRC. We collected data on metabolite perturbations in tumor tissues and body fluids. We sought to elaborate the pathogenetic mechanisms shared by these two cancers and identify those metabolites or mechanisms that were particular to either cancer type. We further sorted different metabolites among the different specimens and provided recommended pools of metabolic biomarkers specific to $\mathrm{GC}$ or CRC for future prospective validation in the early detection and differential diagnosis of these two cancers.

\section{Materials and Methods Literature Search Strategy}

According to PRISMA guidelines, we conducted this metaanalysis (Details of PRISMA checklist for this study were summarized in Table S1). We searched PubMed, Embase, and ScienceDirect to identify all metabolomics studies of GC and CRC published from 1998 through to September 2018. We used various combinations of keywords including "metabolomics", "metabonomics", "colorectal/gastric", "neoplasm/carcinoma/tumor", "NMR", "GC-MS", and "LC-MS". Additionally, a reference list of selected studies was manually examined to avoid missing relevant studies. Two reviewers (JST and NNZ) independently assessed articles for eligibility. Disagreements were resolved by the third reviewer $(\mathrm{FW})$.

\section{Inclusion and Exclusion Criteria}

Studies aiming to find metabolomics characteristics and candidate metabolic biomarkers for diagnosis in GC and $\mathrm{CRC}$ were included. The inclusion criteria were as follows: 1) original studies published in English and available as full texts; 2) studies with a case-control design, nested case-control design, or self-control design (studies detecting tumor tissue using autologous non-tumorous adjacent tissue specimens as matched controls); 3) studies investigating either human tissues, serum, or body fluids; 4) the use of a detection platform including NMR, GC-MS, LCMS, UPLC-MS/MS, HPLC-GC/MS-MS, or multiple platforms; 5) the names of differential metabolites (variable importance in the projection $>1$ and $P<0.05$ ) available for extraction. When duplicated reports were published from 
the same population, the most recent or most complete publication was included. The exclusion criteria were as follows: 1) review articles; 2) abstracts only; 3) animal or cell lines studies; 4) non-English papers; 5) genomics and proteomics research; 6) incomplete data.

\section{Quality Assessment}

We applied QUADOMICS ${ }^{13}$ an adaptation of the quality assessment of diagnostic accuracy assessment (QUADAS), to evaluate the methodological quality of the selected studies. We surmised that use of a cut-off value to assess "omics"-based studies may be inappropriate because of the complexities of new "omics"-based methods. By summarizing the percentage of applied criteria scored positively, QUADOMICS can assess the quality of diagnostic studies in this highly dynamic field. This reduces the challenge of sieving through a huge amount of recently produced data. Two reviewers (JST and NNZ) independently assessed the quality of the included studies. Disagreements were resolved by the third reviewer (FW) or a group meeting.

\section{Data Extraction and Analysis}

The following information was extracted from each study: 1) first author's name and publication year; 2) type of study design; 3) sample size; 4) matching condition; 5) sample source; 6) specimen type; 7) detection platform; 8) altered metabolites (comparing to appropriate control group). Two researchers (JST and NNZ) extracted the information independently. Any disagreement was discussed with the third researcher (FW).

\section{Pathway Enrichment Analysis and Visualization of Specific Pathways} MetaboAnalyst $3.0^{14}$ incorporated with database sources including KEGG (http://www.genome.jp/kegg/) and HMDB (http://www.hmdb.ca/) was used for pathway enrichment analysis. IPath (interactive Pathways Explorer v3), an online analysis tool that can visualize metabolic pathways (http://pathways.embl.de/iPath3.cgi\#), was used to clarify pathways enriched in these two cancers.

\section{Results}

\section{Literature Searching}

The selection process of the eligible studies is presented in Figure S1A (GC) and Figure S1B (CRC). A total of 89 studies (24 articles of GC and 65 articles of CRC) were included in this analysis.

\section{Study Characteristics}

Figure S2A-C) depicts an overview of the studies included in this meta-analysis from three aspects: publication year, epidemiological design, and regions of subjects. There was a similarity in the distribution of study types between GC and CRC. For GC, most studies were case-control studies ( $n=17 ; 70.83 \%)$, with only six (25\%) self-control studies and one $(4.17 \%)$ nested case-control study. Similarly, most of the CRC studies were case-control studies $(n=40$; $61.54 \%)$, with half as many self-control studies ( $n=20$; $30.77 \%)$ and few nested case-control studies $(n=5$; $7.69 \%$ ). Subjects in these studies were from Asia, Europe and North America, with most subjects from Asia.

The specimens and detection platforms for $\mathrm{GC}$ and $\mathrm{CRC}$ studies are depicted in Figure S2D and S2E. There is some diversity in the mode of selection of biological samples and in the chosen detection platforms between the two cancers. Urine and tissue samples were analyzed most in GC studies (Table S2), whereas tissue samples were analyzed most in CRC studies (Table S3). Combinations of multiple platforms were more commonly applied in CRC studies. Among the 24 GC-based studies, 18 studies employed mass spectrometry (MS)-based analytical methods and four studies used NMR. Among the 18 studies, seven used LC-MS, nine used GC-MS and two used CE-MS. Among the 65 CRC-based studies, 40 studies employed MS-based analytical methods (13 LC-MS, 21 GC-MS, 4 CE-MS, 1 FIA-MS and 1 DI-MS), 15 used NMR, and 10 employed multiple platforms.

\section{Quality Assessment}

Most of the studies included in our analysis sufficiently described the details of the clinical and physiological factors pertaining to the cancers $(22 / 24,91.67 \%$ in GC; $55 / 65$, $84.62 \%$ in CRC). More than half of the studies $(20 / 24$, $83.33 \%$ in GC; $43 / 65,66.15 \%$ in CRC) described adequate details in terms of the diagnostic and treatment procedures. All of the studies described the selection criteria clearly and specimen types. Most of the studies $(21 / 24,87.5 \%$ in GC; $58 / 65,89.23 \%$ in CRC) failed to avoid overfitting in the lack of validation sets. Detailed information about questioning items and methodological quality assessment according to QUADOMICS is shown in Table S4 and Table S5.

\section{Differential Metabolites of GC and CRC in Previous Metabolomics Studies}

We extracted 223 altered metabolites in GC patients. Seven amino acids (valine, tyrosine, isoleucine, alanine, 
tryptophan, methionine and leucine) were reported more than five times, and a further 34 metabolites were reported three to five times (Table S6).

Because more studies have been conducted in CRC than GC, a higher number of differential metabolites were reported for CRC. As shown in Table S7, 472 metabolites were altered in CRC patients. Twenty-six differential metabolites (such as glycine, L-valine, L-lactic acid, L-alanine, L-phenylalanine, among others) were reported more than 10 times, 31 metabolites more than five times, and 66 metabolites three to five times. Comparing between the two cancer types, 136 metabolites were shared.

\section{Altered Pathways Enriched by Differential Metabolites Extracted from Single Type of Sample (Blood, Urine, Tissue or Feces)}

We conducted pathway enrichment analyses using metabolites extracted from blood, urine, tissue, or feces (if available), respectively. For GC, there were 27, 14, and 18 pathways enriched by the differential metabolites extracted from tissue, blood, and urine (Table S8). Meanwhile, there were 24, 19, 24, and 18 pathways enriched by the differential metabolites extracted from tissue, blood, urine, and feces, respectively, in CRC (Table S9). As shown in Table 1 and Figure 1, metabolites from tissue covered most of the pathways in both GC and CRC. The number of pathways enriched by urine metabolites was more than that by blood metabolites. Among the GC-related pathways, eight pathways were shared across blood, tissue, and urine (Figure 1A); among the CRCrelated pathways, 11 pathways were shared across blood, urine, feces, and tissue samples (Figure 1C).

\section{Potential Metabolomic Biomarkers Selection Based on Pathways Shared Across Different Sample Types in GC and CRC}

Among the eight pathways shared across blood, tissue, and urine in $\mathrm{GC}, 60$ differential metabolites have been reported in previous studies (eg, tyrosine, taurine, alanine; Table 2). As shown in Figure 1B, 20 metabolites were reproducibly detected in blood and tissue (GC-blood-candidate biomarkers), and 24 metabolites in urine and tissue (GC-urinecandidate biomarkers). Moreover, 12 metabolites were detected in all three specimen types (tissue, blood, and urine), and were thus considered as the best candidate metabolic biomarkers of GC.

For CRC, among the 11 pathways across the different specimen types, 69 differential metabolites were detected (eg, serine, alanine, urea; Table 2). Twenty-three metabolites were reproducibly detected in urine and tissue (CRC-urinecandidate biomarkers), 39 in blood and tissue (CRC-bloodcandidate biomarkers), and 19 in feces and tissue (CRC-fecescandidate biomarkers) (Figure 1D). Thirteen metabolites were shared across all four specimen types, and these were considered as the best candidate metabolic biomarkers of CRC.

\section{Similarity and Dissimilitude of Pathways Between GC and CRC}

We also compared the pathways enriched in multiple specimen types between GC and CRC. Twenty-seven pathways (categorized into eight classifications) were shared between these two cancers, including amino acid metabolism (such as alanine, aspartate and glutamate metabolism, glycine, serine and threonine metabolism, arginine and proline metabolism), carbohydrate metabolism (such as glycolysis or gluconeogenesis, pyruvate metabolism, propanoate metabolism), energy metabolism (nitrogen metabolism, methane metabolism), genetic information processing, lipid metabolism, nucleotide metabolism, metabolism of cofactors and vitamins, and metabolism of other amino acids. A venn diagram shows the common and different pathways between these two cancers (Figure 2).

Strikingly, based on our results, five pathways (nicotinate and nicotinamide metabolism, purine metabolism, D-arginine and D-ornithine metabolism, tryptophan metabolism, and galactose metabolism) were enriched only in GC, whereas eight pathways (tyrosine metabolism, histidine metabolism, pentose and glucuronate interconversions, glycerophospholipid metabolism, fatty acid biosynthesis, primary bile acid biosynthesis, ascorbate and aldarate metabolism, and linoleic acid metabolism) were enriched only in CRC. The typical pathways of GC and CRC are outlined in Figure S3. These metabolic pathways are generally interlaced but specificities can also be observed. In addition, 35 metabolites were included in the five GC pathways, most of which were extracted from blood. Meanwhile, 54 metabolites were included in the eight CRC pathways, of which most were also extracted from blood (Table 3). All these metabolites can be considered as the potential biomarkers for differential diagnosis of these two cancers. 
Table I Pathway Enrichment Analyses Based on the Differential Metabolites of Gastric Cancer and Colorectal Cancer

\begin{tabular}{|c|c|c|c|c|c|c|c|c|}
\hline Pathway Name & Tissue & Blood & Urine & Pathway Name & Tissue & Blood & Urine & Feces \\
\hline Gastric cancer & & & & Colorectal cancer & & & & \\
\hline $\begin{array}{l}\text { Alanine, aspartate and glutamate } \\
\text { metabolism }\end{array}$ & $\sqrt{ }$ & $\sqrt{ }$ & $\sqrt{ }$ & $\begin{array}{l}\text { Alanine, aspartate and glutamate } \\
\text { metabolism }\end{array}$ & $\sqrt{ }$ & $\sqrt{ }$ & $\sqrt{ }$ & $\sqrt{ }$ \\
\hline Aminoacyl-tRNA biosynthesis & $\sqrt{ }$ & $\sqrt{ }$ & $\sqrt{ }$ & Aminoacyl-tRNA biosynthesis & $\sqrt{ }$ & $\sqrt{ }$ & $\sqrt{ }$ & $\sqrt{ }$ \\
\hline Arginine and proline metabolism & $\sqrt{ }$ & $\sqrt{ }$ & $\sqrt{ }$ & Arginine and proline metabolism & $\sqrt{ }$ & $\sqrt{ }$ & $\sqrt{ }$ & $\sqrt{ }$ \\
\hline Nitrogen metabolism & $\sqrt{ }$ & $\sqrt{ }$ & $\sqrt{ }$ & Cyanoamino acid metabolism & $\sqrt{ }$ & $\sqrt{ }$ & $\sqrt{ }$ & $\sqrt{ }$ \\
\hline Phenylalanine metabolism & $\sqrt{ }$ & $\sqrt{ }$ & $\sqrt{ }$ & $\begin{array}{l}\text { Cysteine and methionine } \\
\text { metabolism }\end{array}$ & $\sqrt{ }$ & $\sqrt{ }$ & $\sqrt{ }$ & $\sqrt{ }$ \\
\hline Propanoate metabolism & $\sqrt{ }$ & $\sqrt{ }$ & $\sqrt{ }$ & $\begin{array}{l}\text { Glycine, serine and threonine } \\
\text { metabolism }\end{array}$ & $\sqrt{ }$ & $\sqrt{ }$ & $\sqrt{ }$ & $\sqrt{ }$ \\
\hline Taurine and hypotaurine metabolism & $\sqrt{ }$ & $\sqrt{ }$ & $\sqrt{ }$ & Nitrogen metabolism & $\sqrt{ }$ & $\sqrt{ }$ & $\sqrt{ }$ & $\sqrt{ }$ \\
\hline $\begin{array}{l}\text { Valine, leucine and isoleucine } \\
\text { biosynthesis }\end{array}$ & $\sqrt{ }$ & $\sqrt{ }$ & $\sqrt{ }$ & Pantothenate and CoA biosynthesis & $\sqrt{ }$ & $\sqrt{ }$ & $\sqrt{ }$ & $\sqrt{ }$ \\
\hline $\begin{array}{l}\text { D-Glutamine and D-glutamate } \\
\text { metabolism }\end{array}$ & $\sqrt{ }$ & $\sqrt{ }$ & & Propanoate metabolism & $\sqrt{ }$ & $\sqrt{ }$ & $\sqrt{ }$ & $\sqrt{ }$ \\
\hline $\begin{array}{l}\text { Nicotinate and nicotinamide } \\
\text { metabolism }\end{array}$ & $\sqrt{ }$ & $\sqrt{ }$ & & Taurine and hypotaurine metabolism & $\sqrt{ }$ & $\sqrt{ }$ & $\sqrt{ }$ & $\sqrt{ }$ \\
\hline $\begin{array}{l}\text { D-Arginine and D-ornithine } \\
\text { metabolism }\end{array}$ & & $\sqrt{ }$ & & $\begin{array}{l}\text { Valine, leucine and isoleucine } \\
\text { biosynthesis }\end{array}$ & $\sqrt{ }$ & $\sqrt{ }$ & $\sqrt{ }$ & $\sqrt{ }$ \\
\hline $\begin{array}{l}\text { Phenylalanine, tyrosine and } \\
\text { tryptophan biosynthesis }\end{array}$ & & $\sqrt{ }$ & & Citrate cycle (TCA cycle) & $\sqrt{ }$ & $\sqrt{ }$ & $\sqrt{ }$ & \\
\hline Tryptophan metabolism & & $\sqrt{ }$ & & $\begin{array}{l}\text { Glyoxylate and dicarboxylate } \\
\text { metabolism }\end{array}$ & $\sqrt{ }$ & $\sqrt{ }$ & $\sqrt{ }$ & \\
\hline $\begin{array}{l}\text { Valine, leucine and isoleucine } \\
\text { degradation }\end{array}$ & & $\sqrt{ }$ & & Phenylalanine metabolism & & $\sqrt{ }$ & $\sqrt{ }$ & \\
\hline Beta-Alanine metabolism & $\sqrt{ }$ & & $\sqrt{ }$ & beta-Alanine metabolism & $\sqrt{ }$ & & $\sqrt{ }$ & $\sqrt{ }$ \\
\hline Citrate cycle (TCA cycle) & $\sqrt{ }$ & & $\sqrt{ }$ & Butanoate metabolism & $\sqrt{ }$ & & $\sqrt{ }$ & $\sqrt{ }$ \\
\hline Cyanoamino acid metabolism & $\sqrt{ }$ & & $\sqrt{ }$ & Glutathione metabolism & $\sqrt{ }$ & & $\sqrt{ }$ & $\sqrt{ }$ \\
\hline $\begin{array}{l}\text { Glycine, serine and threonine } \\
\text { metabolism }\end{array}$ & $\sqrt{ }$ & & $\sqrt{ }$ & Sulfur metabolism & $\sqrt{ }$ & & $\sqrt{ }$ & $\sqrt{ }$ \\
\hline $\begin{array}{l}\text { Glyoxylate and dicarboxylate } \\
\text { metabolism }\end{array}$ & $\sqrt{ }$ & & $\sqrt{ }$ & $\begin{array}{l}\text { D-Glutamine and D-glutamate } \\
\text { metabolism }\end{array}$ & & & $\sqrt{ }$ & $\sqrt{ }$ \\
\hline Methane metabolism & $\sqrt{ }$ & & $\sqrt{ }$ & $\begin{array}{l}\text { Valine, leucine and isoleucine } \\
\text { degradation }\end{array}$ & & & $\sqrt{ }$ & $\sqrt{ }$ \\
\hline Pantothenate and CoA biosynthesis & $\sqrt{ }$ & & $\sqrt{ }$ & Pyrimidine metabolism & $\sqrt{ }$ & & $\sqrt{ }$ & \\
\hline Pyrimidine metabolism & $\sqrt{ }$ & & $\sqrt{ }$ & Histidine metabolism & & & $\sqrt{ }$ & \\
\hline Pyruvate metabolism & $\sqrt{ }$ & & $\sqrt{ }$ & $\begin{array}{l}\text { Phenylalanine, tyrosine and } \\
\text { tryptophan biosynthesis }\end{array}$ & & & $\sqrt{ }$ & \\
\hline Purine metabolism & & & $\sqrt{ }$ & Tyrosine metabolism & & & $\sqrt{ }$ & \\
\hline Butanoate metabolism & $\sqrt{ }$ & & & Ascorbate and aldarate metabolism & & $\sqrt{ }$ & & \\
\hline $\begin{array}{l}\text { Cysteine and methionine } \\
\text { metabolism }\end{array}$ & $\sqrt{ }$ & & & Glycerophospholipid metabolism & & $\sqrt{ }$ & & \\
\hline Galactose metabolism & $\sqrt{ }$ & & & Linoleic acid metabolism & & $\sqrt{ }$ & & \\
\hline Glutathione metabolism & $\sqrt{ }$ & & & $\begin{array}{l}\text { Pentose and glucuronate } \\
\text { interconversions }\end{array}$ & & $\sqrt{ }$ & & \\
\hline Glycolysis or Gluconeogenesis & $\sqrt{ }$ & & & Primary bile acid biosynthesis & & $\sqrt{ }$ & & \\
\hline Pentose phosphate pathway & $\sqrt{ }$ & & & Glycolysis or Gluconeogenesis & $\sqrt{ }$ & & & $\sqrt{ }$ \\
\hline Sulfur metabolism & $\sqrt{ }$ & & & Fatty acid biosynthesis & $\sqrt{ }$ & & & \\
\hline $\begin{array}{l}\text { Synthesis and degradation of ketone } \\
\text { bodies }\end{array}$ & $\sqrt{ }$ & & & Methane metabolism & $\sqrt{ }$ & & & \\
\hline
\end{tabular}


Table I (Continued).

\begin{tabular}{|c|c|c|c|c|c|c|c|c|}
\hline Pathway Name & Tissue & Blood & Urine & Pathway Name & Tissue & Blood & Urine & Feces \\
\hline & & & & $\begin{array}{l}\text { Pentose phosphate pathway } \\
\text { Purine metabolism } \\
\text { Pyruvate metabolism } \\
\text { Synthesis and degradation of ketone } \\
\text { bodies }\end{array}$ & $\begin{array}{l}\sqrt{ } \\
\sqrt{ } \\
\sqrt{ } \\
\sqrt{ }\end{array}$ & & & \\
\hline
\end{tabular}

\section{Discussion}

Metabolic profiles provide a top-down "systems level" detail of an organism under a particular physiological status, which fluctuates according to pathological demands. ${ }^{15,16}$ Numerous studies have sought to identify changes in metabolite levels, and this has resulted in the rapid increase in biomarker discovery, particularly for GC and CRC.

Some criteria for developing and assessing biomarkers are necessary, such as good repeatability of high

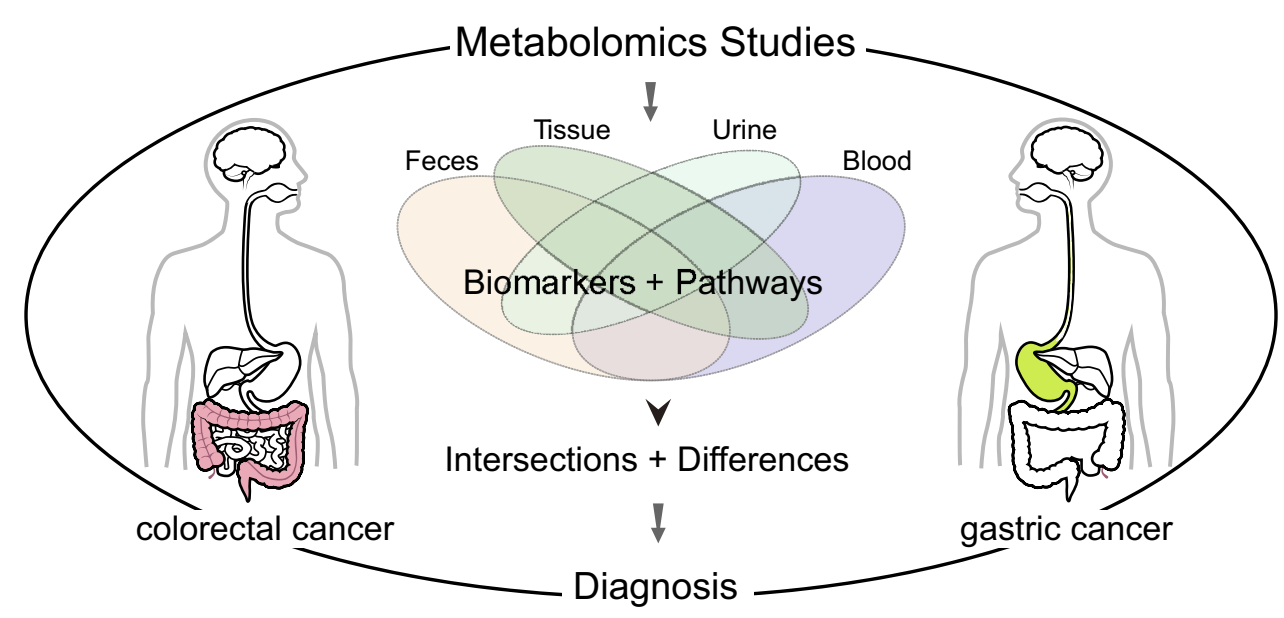

Graphical abstract

A

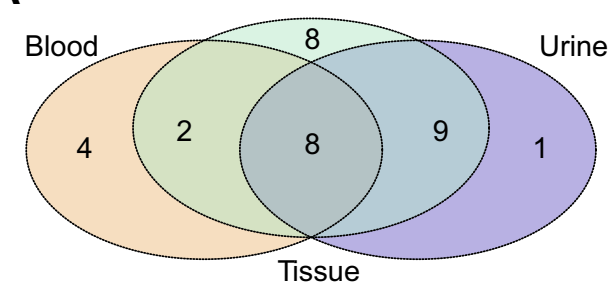

C

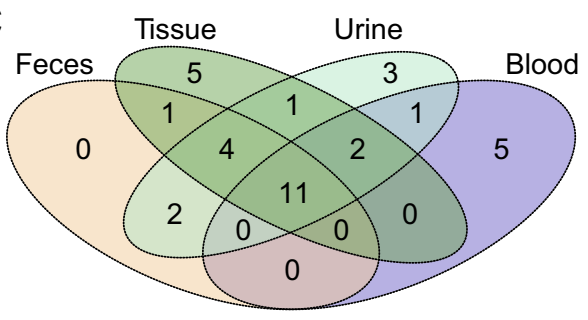

B

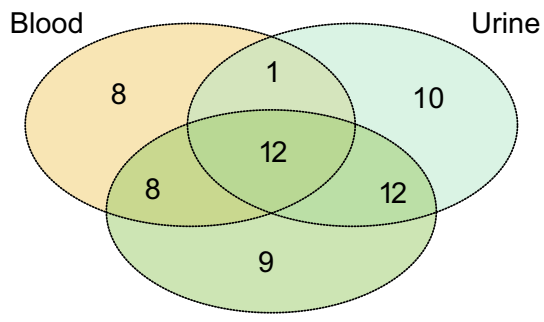

Tissue

D

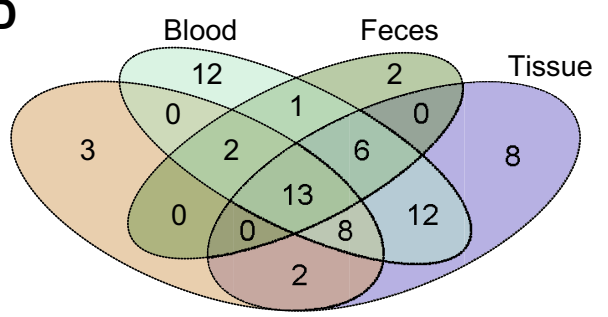

Figure I Pathway and metabolite distributions among different types of specimens. (A) Pathway enrichment analyses of GC based on the extracted metabolites from different types of specimens. (B) Metabolites (enriched in eight common pathways) in different types of specimens in GC. (C) Pathway enrichment analyses of CRC based on the extracted metabolites from different types of specimens. (D) Metabolites (enriched in the II common pathways) in different types of specimens. 


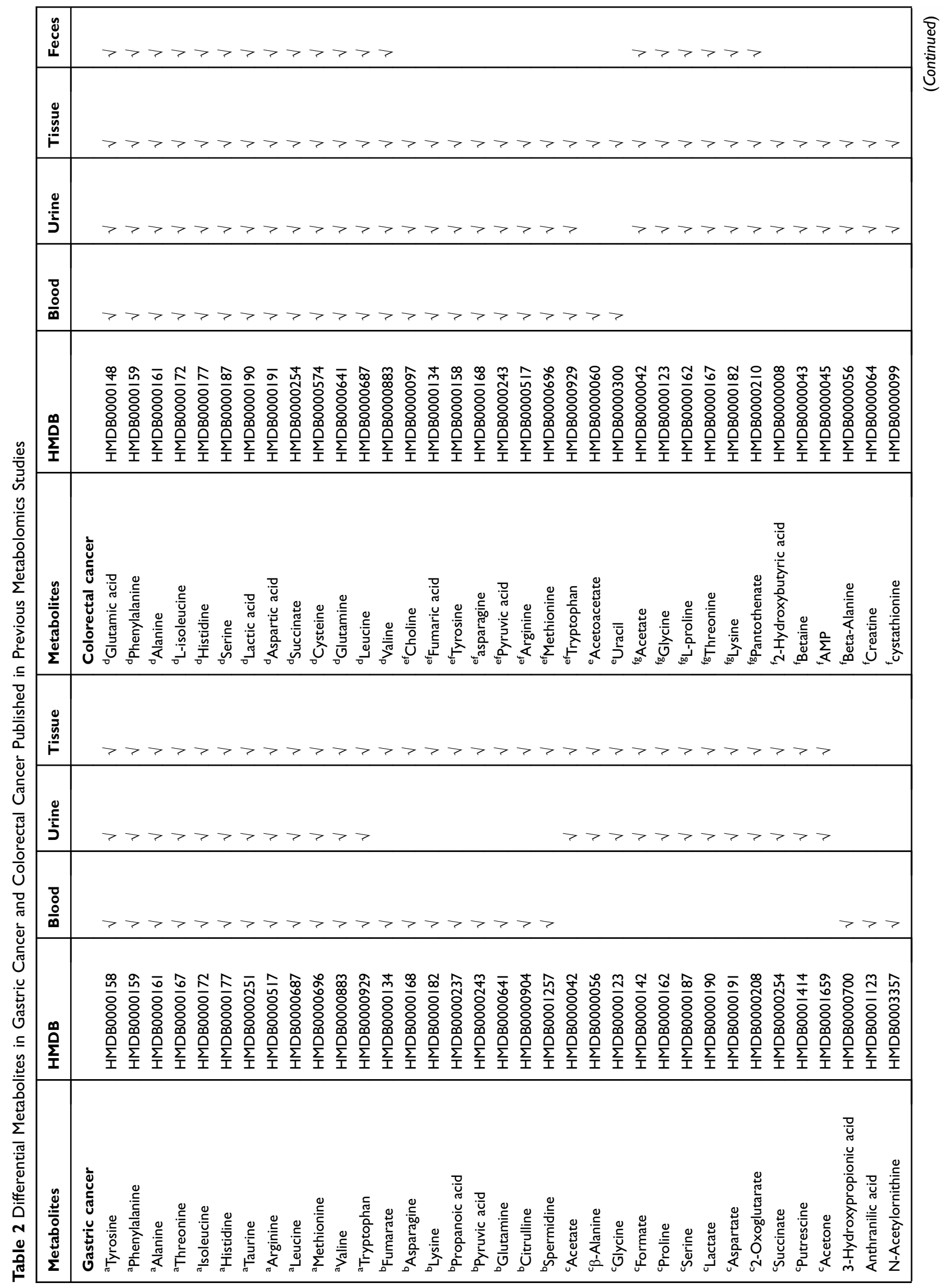




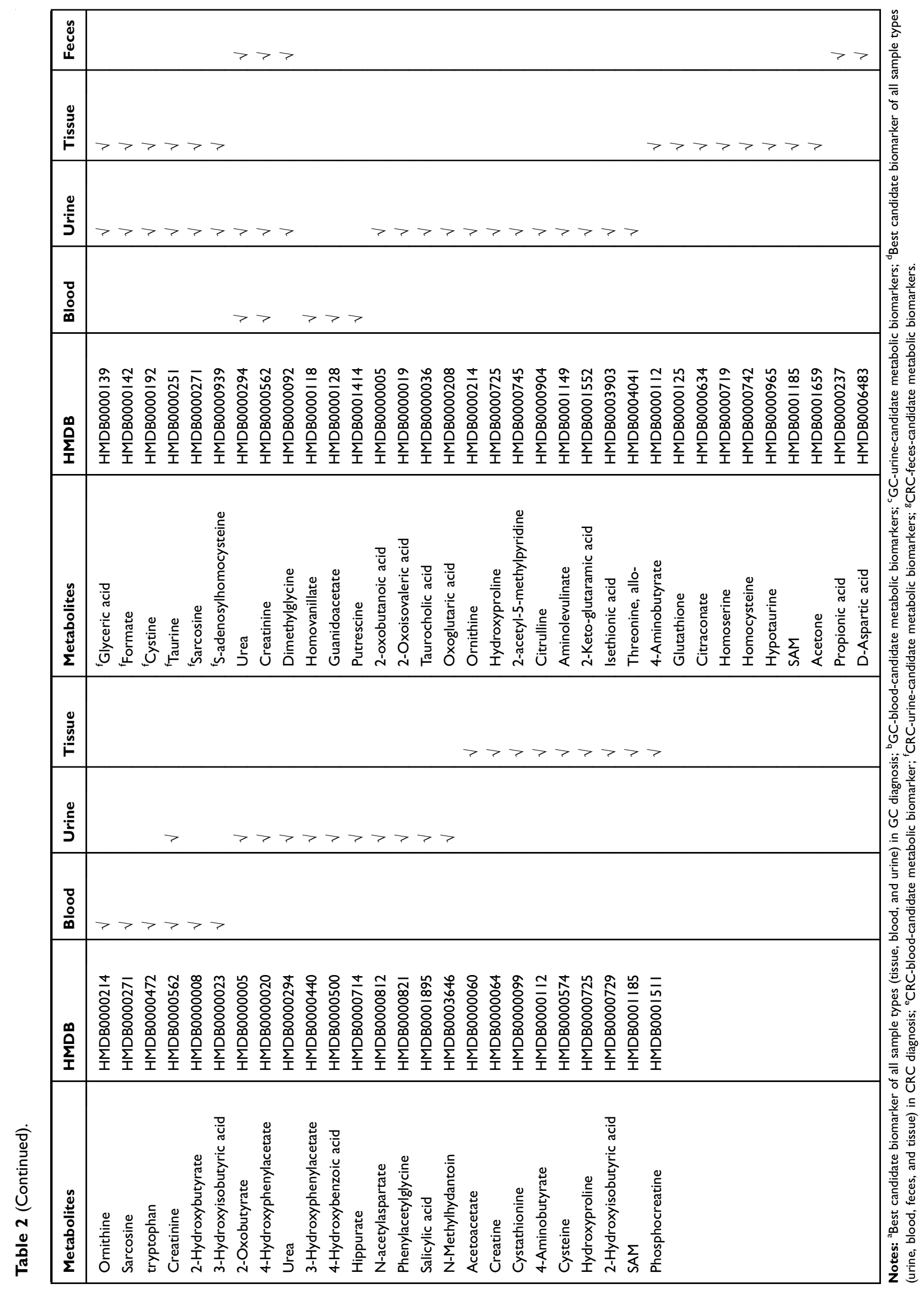




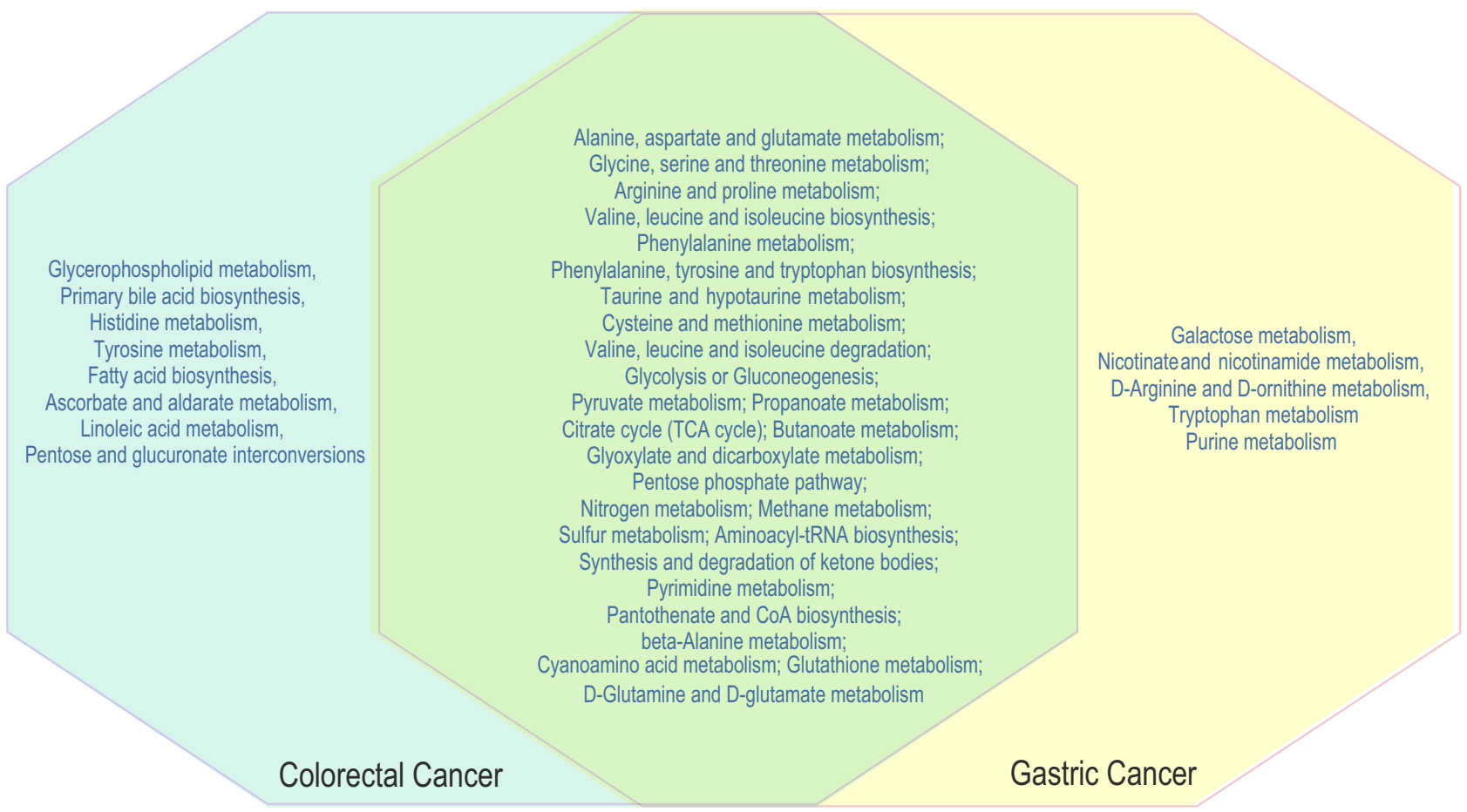

Figure 2 Similarities and differences in metabolic pathways between gastric cancer and colorectal cancer.

sensitivity and high specificity, reproducible validation in multiple populations, and prospective evaluation in cohort population, etc. However, current identified metabolic biomarkers have several limitations, including unstandardized protocols for sample acquisition and preparation in different studies; most markers were identified in single population by a single cross-sectional comparison of case-control samples. All factors described above induce the challenge of enormous number and inconsistency of reported biomarkers, and consequently cause the difficulty in prospective validation. Moreover, one of the greatest challenges in metabolic biomarker research is to identify markers that can accurately distinguish among different cancers, particularly where there are overlapping metabolic alterations and phenotypes. This study provides a systematic review of all previously published metabolomics studies of GC and CRC. A key contribution of our work is the provision of recommend candidate metabolic biomarkers of $\mathrm{GC}$ and $\mathrm{CRC}$ for future validation in clinic diagnosis. By comparing the metabolites extracted from previous studies, it is possible that substances that are repeated in different detection platforms, different biological samples, and multiple populations to be identified. Especially, the metabolites repeatedly detected in tumor tissue and biological fluids have the potential as candidate biomarkers. Besides, the results of pathway enrichment have implications for the further discovery of mechanisms in GC and CRC and help us to understand the disease occurrences.

\section{Similarity of Metabolic Pathways Between GC and CRC}

Different cancers can share common metabolic features, such as the deregulated uptake of glucose and amino acids, the increased demand for nitrogen, and the use of glycolysis/ TCA cycle intermediates for biosynthesis and NADPH production. ${ }^{17}$ Some studies have shown that gastrointestinal cancers display similar mechanisms. ${ }^{18,19}$ In this study, we observed that nearly half of the altered metabolites were shared across GC and CRC, as well as same altered mechanisms, including changes in carbohydrate, and nucleotide metabolism, nitrogen metabolism and amino acid metabolism.

In both $\mathrm{GC}$ and $\mathrm{CRC}$, we identified alterations in pyruvic acid, malic acid, succinate, fumarate, citrate, isocitric acid, alpha-ketoglutaric acid, L-glutamine, beta-alanine and 3-aminoisobutanoic acid, which involved in carbohydrate and nucleotide metabolism. Cancer cells exhibit an increased dependence on the glycolytic pathway for ATP generation, ${ }^{20}$ which is called the Warburg effect. This phenomenon is characterized by increased aerobic glycolysis, an increased production of lactic acid, and impaired DNA repair 


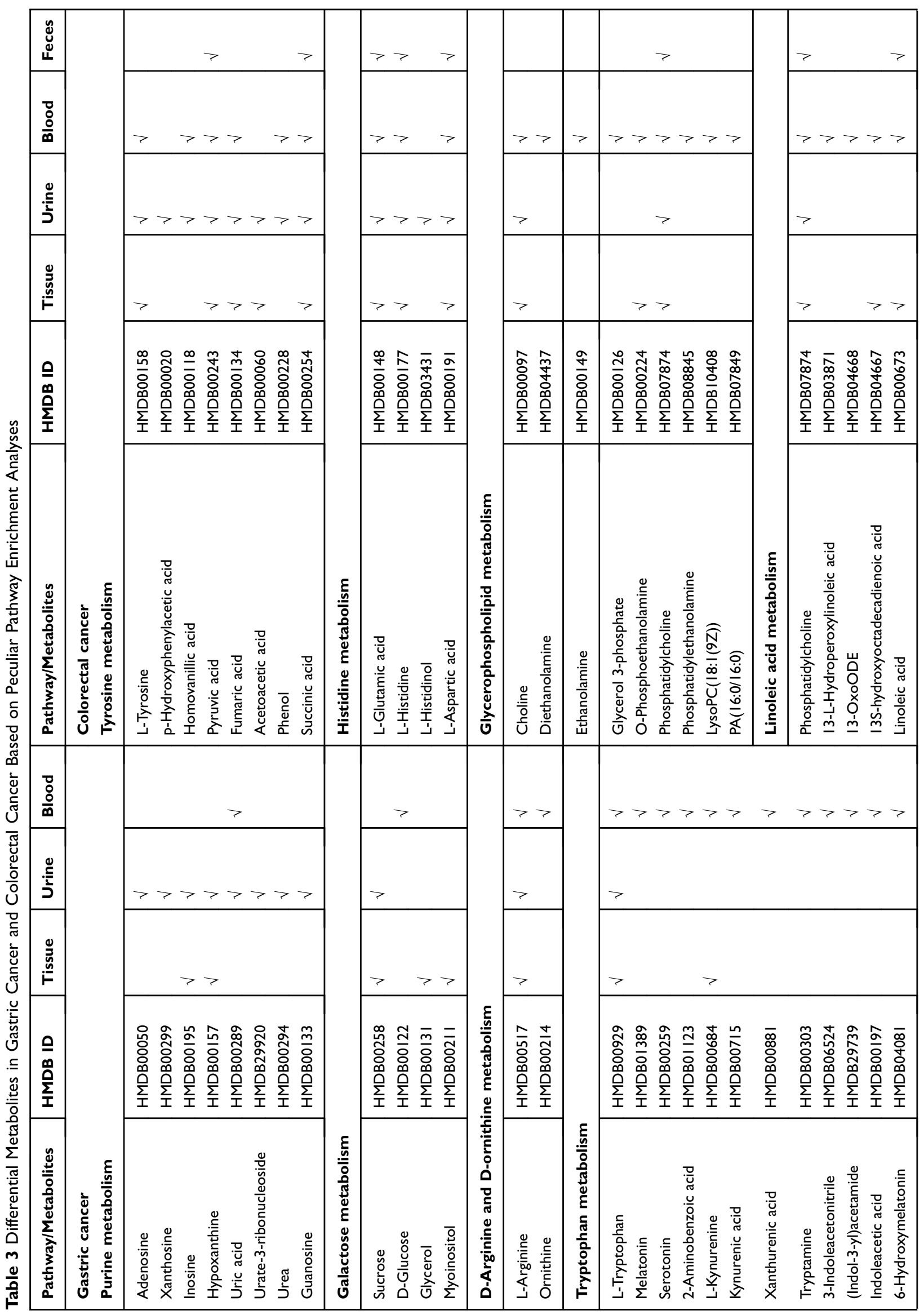




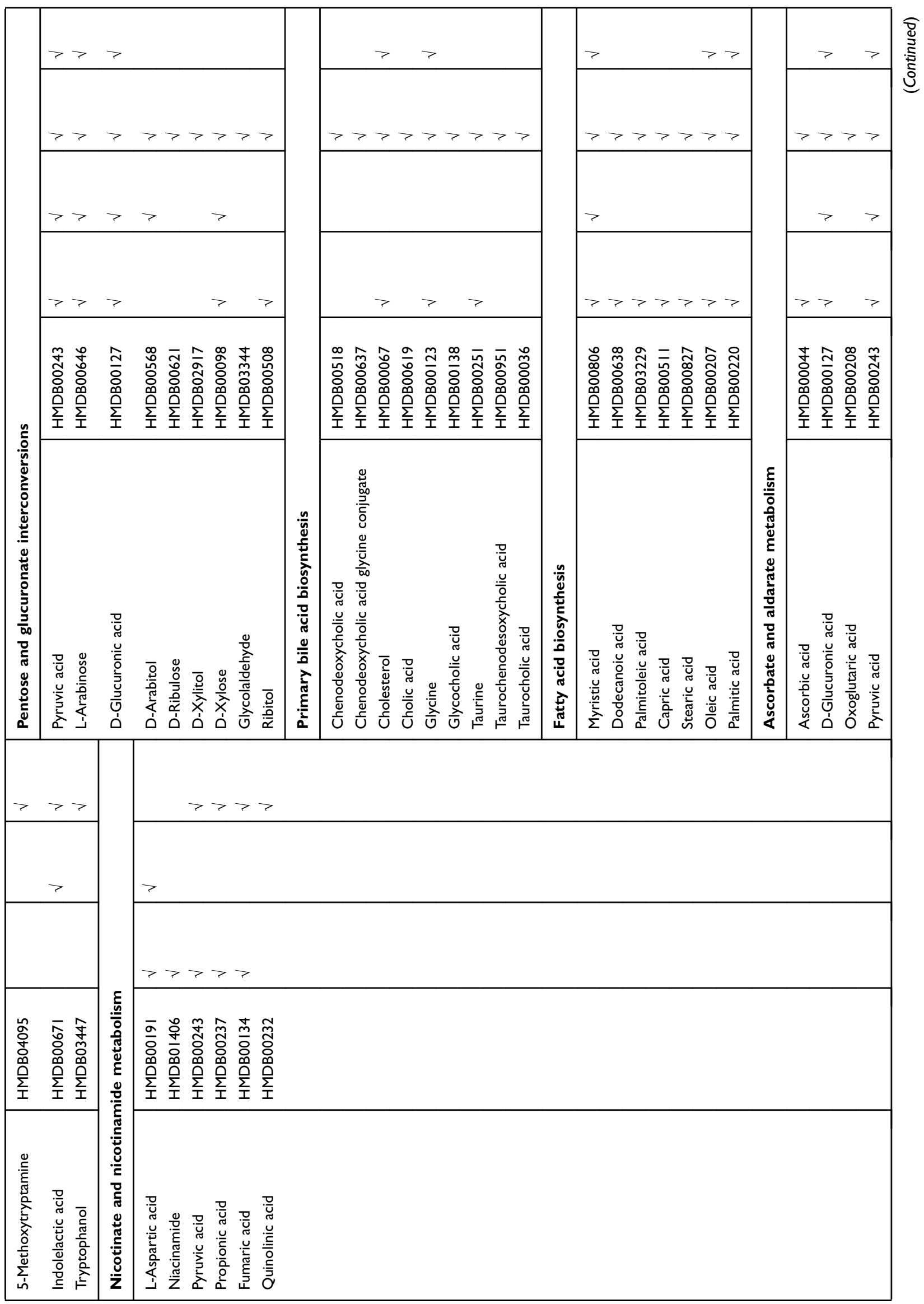




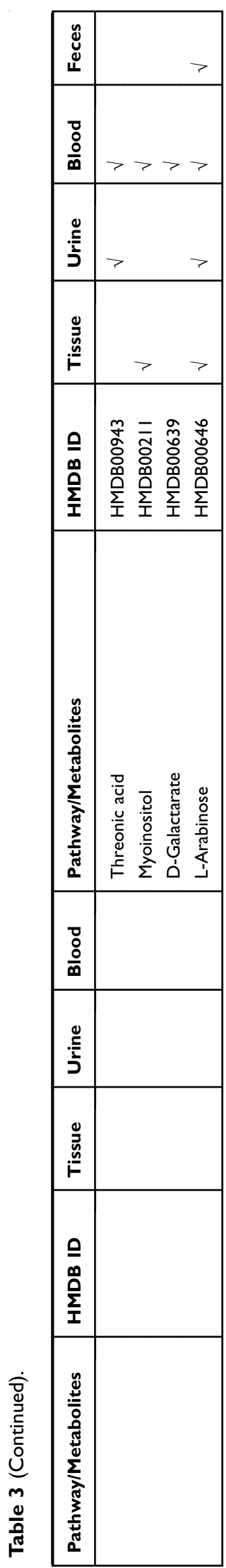

mechanisms. ${ }^{21}$ The abnormal changes in carbohydrate and nucleotide metabolism demonstrate this effect. Nucleotides are necessary for numerous cellular processes, with imbalances in nucleotide levels linked with various human diseases, including cancer. ${ }^{22}$ Early studies in yeast models showed that decreased dNTP levels lead to increased mutagenesis through an increase in genomic instability. ${ }^{23}$

Nitric oxide is a signalling molecule in nitrogen metabolism, and its roles in cancer formation, progression, and metastasis have been extensively investigated. ${ }^{24}$ The activation of nitric oxide synthase (NOS) and elevated nitric oxide levels have antitumor effects, ${ }^{25}$ however, nitric oxide may also promote cancer formation and progression. ${ }^{26}$ Therefore, the effect of nitric oxide on metastasis is likely associated with other factors, such as cell type, drug dosage, the affected organ, and perhaps the stage of metastasis. ${ }^{27,28}$ Cianchi et $\mathrm{al}^{29}$ previously showed that nitric oxide stimulates COX-2 activity in CRC. Others have also linked inflammation to carcinogenesis. Gastric mucosal inflammation in response to Helicobacter pylori infection results in an upregulation of interleukin-1beta (IL-1 $\beta$ ) and an overproduction of mutagenic nitric oxide. These two factors induce aberrant DNA methylation, which can lead to carcinogenesis. ${ }^{30}$

Methane is mainly produced by Methanobrevibacter smithii ${ }^{31}$ through the conversion of $\mathrm{H}_{2}$ generated by gut microflora in the presence of specific microbes. Evidence shows that methane production is associated with gastrointestinal conditions, with greater methane production observed in $H$. pylori-infected patients than in noninfected patients ${ }^{32} H$. pylori infection is associated with changes in the gastric microenvironment, which in turn affect the gastric microbiota composition and can trigger changes in the large intestinal microbiota. ${ }^{33}$ There is some evidence that $H$. pylori is an essential factor during the formation of gastric cancer. ${ }^{34} H$. pylori infection is suggested to increase the secretion of gastrin, which may lead alterations in the mucosal cells of the colorectum. ${ }^{35,36}$

\section{Dissimilitudes of Metabolic Pathways Between GC and CRC}

Eight pathways were only enriched in CRC (Figure 3). One such pathway was glycerophospholipid metabolism. Fat digestion begins in the upper intestine through the action of various enzymes and bile salts. Lipids, such as triglycerides, phospholipids, cholesterol, and glycolipids, play important roles in maintaining the normal 
physiological functions of cells, the integrity of cellular barriers and membrane matrices, in signalling, and as a source of energy. ${ }^{37,38}$ Abnormal lipid metabolism occurs in cancer, ${ }^{39}$ and manifests as disruptions in cell proliferation, apoptosis, immunity, angiogenesis, and inflammation. ${ }^{38,40}$ During fatty acid metabolism, clostridia - part of the human flora - can accelerate the transformation of secondary bile acid, which can act as a carcinogenic substance and promote CRC. ${ }^{41}$ Others have reported reduced levels of lysophosphatidylcholine (lysoPC) in tumor cells. ${ }^{42,43}$ Raynor et al found the metastatic tumor cells can hydrolyse lysoPC extracellularly to glyceroPC and free fatty acids. This production of free fatty acids satisfies the tumor cell's high energy demands while maintaining membrane fluidity and aiding in the generation of pro-metastatic lipid second messengers. ${ }^{44}$ Interactions between fatty acids, bile acids, and intestinal flora can specifically produce diacylglycerol, prostaglandins, and leukotrienes, leading to tumorigenesis by activating immune or inflammatory responses in CRC. ${ }^{45,46}$

Bile acids cause DNA damage ${ }^{47}$ and have been suggested to be promoters of colon carcinogenesis. ${ }^{48}$ High-fat diets can promote the hepatic synthesis of bile acids and increase their delivery to the colonic lumen. After secretion to the intestinal lumen, primary bile acids are deconjugated by microbial bile salt hydrolases and most bile acids are reabsorbed by passive diffusion and active transport during small intestinal transit. The remaining bile acids enter the colon, where they undergo extensive biotransformation mediated by colonic bacteria. High-fat diets stimulate the growth and activity of $7 \alpha-$ dehydroxylating bacteria, which convert primary bile acids into secondary bile acids, which are associated with tumorigenic activity. ${ }^{49}$ High concentrations of secondary bile acids in the feces, blood, and bile have been linked to the pathogenesis of cholesterol gallstone disease and colon cancer. $^{50}$

In this study, five pathways were found associated only with GC (Figure 3). Tryptophan is one of the most essential amino acids, which can be metabolized to kynurenic acid, picolinic acid and $\mathrm{NAD}^{+}$through the kynurenine pathway, a major pathway in tryptophan metabolism. ${ }^{51}$ Studies have shown that tryptophan metabolism is involved in evading immune surveillance in cancer. ${ }^{52}$ Studies have also indicated that gastric tumor cells may promote immune escape via indoleamine 2, 3 dioxygenase 1 (IDO1) activity. ${ }^{53}$ IDO1 is the first and rate-limiting step in the kynurenic pathway. Overexpression of IDO1 therefore leads to tryptophan deficiency, affecting the proliferation and differentiation of $\mathrm{T}$ cells, NK cells and other cells, which are eventually cleared because of apoptosis.

\section{Implications for the Exploration and Application of Metabolic Biomarkers}

For solid tumors, tissue samples usually provide the most complete metabolic changes that occur during tumor development and reveal the possible pathophysiological mechanisms. However, blood metabolite profiles show less diurnal variation and less inter- and intra-subject variability. ${ }^{54}$ Urine as a biological waste material, is sterile, easy to obtain in large volumes, mostly free from interfering proteins or lipids, and chemically complex; thus, it has long been a "favoured" biofluid in various global metabolomics studies. Feces and urine have similar biological characteristics: both are involved in the elimination of waste accumulation in the body and can be completely noninvasive during sample collection. However, urine and feces contain metabolic breakdown products from a wide range of food, drink, drugs and environmental contaminants. As observed in this study, more pathways and metabolites were altered in feces and urine than in blood. Special attention should be paid to the biological significance and tumor tissue specificity of biomarkers from feces and urine, because not all markers reflect the specific metabolic characteristics of the cancer. Thus, metabolites enriched in cancer biological pathways and replicated in target tissues and organs have more tumor specificity and a higher potential to be a candidate diagnostic biomarker.

In our study, 8 pathways in GC and 11 pathways in CRC were significantly enriched in all specimens, which indicate that these pathways show the most extensive metabolic changes in GC and CRC cancer patients, and represent the typical characteristics of cancer development. Moreover, we summarized the metabolites that have been duplicated detected in blood/urine/feces and tissue, which can be seen as the pools of candidate metabolites of GC or CRC. Especially, among these pathways, there were 12 metabolites in GC and 13 metabolites in CRC detected across all specimens, indicating their potential utility as candidate diagnostic biomarkers in multiple types of sample. Other researchers may choose a combination of markers according to their actual needs and design prospective studies based on our summarization.

All the pathways shared between GC and CRC in our analysis revealed the typical metabolic alterations seen in 
gastrointestinal cancer. The pathways particularly enriched in GC or CRC are characteristic of the specific metabolic alterations in each cancer. Identifying specific metabolites in these discrete pathways could improve the discrimination ability of GC and CRC in diagnosis (Table 3); for example, using biomarkers in glycerophospholipid metabolism and fatty acid biosynthesis as a diagnostic for CRC.

The current study has some limitations. Although we comprehensively collected original literatures, publication bias is still hard to avoid. Most of the included studies are non-target metabolomics analysis; several factors may influence the results of untargeted metabolic profiling analyses, such as unstandardized protocols for sample acquisition and preparation, and the use of different analytical platforms with various strengths of coverage, sensitivity, and selectivity for chemical classes $^{55,56}$ in different studies. In addition, only the names of differential metabolites could be extracted from original literatures, we cannot evaluate the diagnostic value of each candidate metabolic biomarker using ROC (Receiver Operating Characteristic Curve) analysis. Future studies in multiple populations of these two cancers, using a standardized protocol and platform(s) with quantitative detection of metabolites are needed to confirm our conclusions in this systematic review.

\section{Conclusions}

In this review, pathways shared between or particularly enriched in GC and CRC revealed the similarities and specificities of occurrence and development in these two cancers. Metabolites that have been duplicated detected in multiple types of sample in GC and CRC indicated their potential utility as candidate diagnostic biomarkers. Moreover, identifying specific metabolites in pathways peculiar to GC and CRC could improve the discrimination ability in diagnosis. Our proposed metabolic biomarkers based on this systematic review will have important implications for prospective validation of GC and CRC.

\section{Author Contributions}

Fan Wang and Maoqing Wang contributed to the concept, design and interpretation of data. Jingshen Tian drafted the manuscript. All authors contributed to the acquisition, analysis, and interpretation of data. All authors took part in drafting, revising or critically reviewing the article. All authors have given final approval of the version to be published, and agree to be accountable for all aspects of the work.

\section{Funding}

This work was supported by grants from National Natural Science Foundation of China $(81773503,81573147)$, Scientific Research Foundation for the Returned Overseas Scholars of Heilongjiang Province (LC2018033), and Dr. Wu Lien-teh Science Foundation of Harbin Medical University (WLD-QN1106).

\section{Disclosure}

The authors report no conflicts of interest in this work.

\section{References}

1. Siegel RL, Miller KD, Jemal A. Cancer statistics, 2018. CA Cancer J Clin. 2018;68(1):7-30. doi:10.3322/caac.21442

2. Siegel RL, Miller KD, Fedewa SA, et al. Colorectal cancer statistics, 2017. CA Cancer J Clin. 2017;67(3):177-193. doi:10.3322/ caac. 21395

3. Siegel RL, Miller KD, Jemal A. Cancer statistics, 2017. CA Cancer J Clin. 2017;67(1):7-30. doi:10.3322/caac.21387

4. Cunningham D, Atkin W, Lenz HJ, et al. Colorectal cancer. Lancet. 2010;375(9719):1030-1047. doi:10.1016/S0140-6736(10)60353-4

5. Board PDQATE. Anal cancer treatment (PDQ(R)): health professional version. In: $P D Q$ Cancer Information Summaries. Bethesda (MD): National Cancer Institute (US); 2002.

6. Chan AW, Gill RS, Schiller D, Sawyer MB. Potential role of metabolomics in diagnosis and surveillance of gastric cancer. World J Gastroenterol. 2014;20(36):12874-12882. doi:10.3748/wjg.v20. i36.12874

7. Deng K, Lin S, Zhou L, et al. Three aromatic amino acids in gastric juice as potential biomarkers for gastric malignancies. Anal Chim Acta. 2011;694(1-2):100-107. doi:10.1016/j.aca.2011.03.053

8. Wanebo HJ, Rao B, Pinsky CM, et al. Preoperative carcinoembryonic antigen level as a prognostic indicator in colorectal cancer. $N$ Engl J Med. 1978;299(9):448-451. doi:10.1056/NEJM197808312990904

9. Ni Y, Xie G, Jia W. Metabonomics of human colorectal cancer: new approaches for early diagnosis and biomarker discovery. J Proteome Res. 2014;13(9):3857-3870. doi:10.1021/pr500443c

10. Zhu J, Djukovic D, Deng L, et al. Colorectal cancer detection using targeted serum metabolic profiling. J Proteome Res. 2014;13 (9):4120-4130. doi:10.1021/pr500494u

11. Wu H, Xue R, Tang Z, et al. Metabolomic investigation of gastric cancer tissue using gas chromatography/mass spectrometry. Anal Bioanal Chem. 2010;396(4):1385-1395. doi:10.1007/s00216-0093317-4

12. Spratlin JL, Serkova NJ, Eckhardt SG. Clinical applications of metabolomics in oncology: a review. Clin Cancer Res. 2009;15 (2):431-440. doi:10.1158/1078-0432.CCR-08-1059

13. Lumbreras B, Porta M, Marquez S, Pollan M, Parker LA, HernandezAguado I. QUADOMICS: an adaptation of the quality assessment of diagnostic accuracy assessment (QUADAS) for the evaluation of the methodological quality of studies on the diagnostic accuracy of 'omics'-based technologies. Clin Biochem. 2008;41(16-17):1316-1325. doi:10.1016/j.clinbiochem.2008.06.018

14. Xia J, Sinelnikov IV, Han B, Wishart DS. MetaboAnalyst 3.0-making metabolomics more meaningful. Nucleic Acids Res. 2015;43(W1): W251-257. doi:10.1093/nar/gkv380

15. Liesenfeld DB, Habermann N, Owen RW, Scalbert A, Ulrich CM. Review of mass spectrometry-based metabolomics in cancer research. Cancer Epidemiol Biomarkers Prev. 2013;22 (12):2182-2201. doi:10.1158/1055-9965.EPI-13-0584 
16. Johnson CH, Ivanisevic J, Siuzdak G. Metabolomics: beyond biomarkers and towards mechanisms. Nat Rev Mol Cell Biol. 2016;17 (7):451-459. doi:10.1038/nrm.2016.25

17. Pavlova NN, Thompson CB. The emerging hallmarks of cancer metabolism. Cell Metab. 2016;23(1):27-47. doi:10.1016/j. cmet.2015.12.006

18. Liu L, Chen F, Xiu A, Du B, Ai H, Xie W. Identification of key candidate genes and pathways in endometrial cancer by integrated bioinformatical analysis. Asian Pac J Cancer Prev. 2018;19 (4):969-975. doi:10.22034/APJCP.2018.19.4.969

19. Garrett WS. Cancer and the microbiota. Science. 2015;348 (6230):80-86. doi:10.1126/science.aaa4972

20. Alfarouk KO. Tumor metabolism, cancer cell transporters, and microenvironmental resistance. J Enzyme Inhib Med Chem. 2016;31 (6):859-866. doi:10.3109/14756366.2016.1140753

21. Vander Heiden MG, Cantley LC, Thompson CB. Understanding the Warburg effect: the metabolic requirements of cell proliferation. Science. 2009;324(5930):1029-1033. doi:10.1126/science.1160809

22. Chabosseau P, Buhagiar-Labarchede G, Onclercq-Delic R, et al Pyrimidine pool imbalance induced by BLM helicase deficiency contributes to genetic instability in Bloom syndrome. Nat Commun. 2011;2:368. doi:10.1038/ncomms 1363

23. Holmberg C, Fleck O, Hansen HA, et al. Ddb1 controls genome stability and meiosis in fission yeast. Genes Dev. 2005;19 (7):853-862. doi:10.1101/gad.329905

24. Williams EL, Djamgoz MB. Nitric oxide and metastatic cell behaviour. Bioessays. 2005;27(12):1228-1238. doi:10.1002/ bies. 20324

25. Xie K, Dong Z, Fidler IJ. Activation of nitric oxide synthase gene for inhibition of cancer metastasis. J Leukoc Biol. 1996;59(6):797-803. doi:10.1002/jlb.59.6.797

26. Bing RJ, Miyataka M, Rich KA, et al. Nitric oxide, prostanoids, cyclooxygenase, and angiogenesis in colon and breast cancer. Clin Cancer Res. 2001;7(11):3385-3392.

27. Le X, Wei D, Huang S, Lancaster JR, Xie K. Nitric oxide synthase II suppresses the growth and metastasis of human cancer regardless of its up-regulation of protumor factors. Proc Natl Acad Sci U S A. 2005;102(24):8758-8763. doi:10.1073/pnas.0409581102

28. Ishikawa T, Yoshida N, Higashihara H, et al. Different effects of constitutive nitric oxide synthase and heme oxygenase on pulmonary or liver metastasis of colon cancer in mice. Clin Exp Metastasis. 2003;20(5):445-450. doi:10.1023/A:1025448403124

29. Cianchi F, Cortesini C, Fantappie O, et al. Cyclooxygenase-2 activation mediates the proangiogenic effect of nitric oxide in colorectal cancer. Clin Cancer Res. 2004;10(8):2694-2704. doi:10.1158/10780432.CCR-03-0192

30. Huang FY, Chan AO, Rashid A, Wong DK, Cho CH, Yuen MF. Helicobacter pylori induces promoter methylation of E-cadherin via interleukin-1beta activation of nitric oxide production in gastric cancer cells. Cancer. 2012;118(20):4969-4980. doi:10.1002/cncr.27519

31. Triantafyllou K, Chang C, Pimentel M. Methanogens, methane and gastrointestinal motility. J Neurogastroenterol Motil. 2014;20 (1):31-40. doi:10.5056/jnm.2014.20.1.31

32. Del Zompo F, Ojetti V, Feliciani D, et al. Helicobacter pylori infection is associated with high methane production during lactulose breath test. Eur Rev Med Pharmacol Sci. 2016;20(16):3452-3456.

33. Lopetuso LR, Scaldaferri F, Franceschi F, Gasbarrini A. The gastrointestinal microbiome - functional interference between stomach and intestine. Best Pract Res Clin Gastroenterol. 2014;28(6):995-1002. doi:10.1016/j.bpg.2014.10.004

34. Huang JQ, Zheng GF, Sumanac K, Irvine EJ, Hunt RH. Metaanalysis of the relationship between cagA seropositivity and gastric cancer. Gastroenterology. 2003;125(6):1636-1644. doi:10.1053/j. gastro.2003.08.033
35. Machida-Montani A, Sasazuki S, Inoue M, et al. Atrophic gastritis, Helicobacter pylori, and colorectal cancer risk: a case-control study. Helicobacter. 2007;12(4):328-332. doi:10.1111/j.15235378.2007.00513.x

36. Inoue I, Mukoubayashi C, Yoshimura N, et al. Elevated risk of colorectal adenoma with Helicobacter pylori-related chronic gastritis: a population-based case-control study. Int J Cancer. 2011;129 (11):2704-2711. doi:10.1002/ijc.25931

37. Huse M, Le Floc'h A, Liu X. From lipid second messengers to molecular motors: microtubule-organizing center reorientation in $\mathrm{T}$ cells. Immunol Rev. 2013;256(1):95-106. doi:10.1111/imr.12116

38. Wang M, Wang C, Han RH, Han X. Novel advances in shotgun lipidomics for biology and medicine. Prog Lipid Res. 2016;61:83-108. doi:10.1016/j.plipres.2015.12.002

39. Wenk MR. The emerging field of lipidomics. Nat Rev Drug Discov. 2005;4(7):594-610. doi:10.1038/nrd1776

40. Huse M. Lipid-based patterning of the immunological synapse. Biochem Soc Trans. 2014;42(6):1506-1511. doi:10.1042/ BST20140191

41. Farhana L, Nangia-Makker P, Arbit E, et al. Bile acid: a potential inducer of colon cancer stem cells. Stem Cell Res Ther. 2016;7 (1):181. doi:10.1186/s13287-016-0439-4

42. Taylor LA, Arends J, Hodina AK, Unger C, Massing U. Plasma lyso-phosphatidylcholine concentration is decreased in cancer patients with weight loss and activated inflammatory status. Lipids Health Dis. 2007;6:17. doi:10.1186/1476-511X-6-17

43. Zhao Z, Xiao Y, Elson P, et al. Plasma lysophosphatidylcholine levels: potential biomarkers for colorectal cancer. J Clin Oncol. 2007;25(19):2696-2701. doi:10.1200/JCO.2006.08.5571

44. Raynor A, Jantscheff P, Ross T, et al. Saturated and mono-unsaturated lysophosphatidylcholine metabolism in tumour cells: a potential therapeutic target for preventing metastases. Lipids Health Dis. 2015;14:69. doi:10.1186/s12944-015-0070-x

45. Savari S, Vinnakota K, Zhang Y, Sjolander A. Cysteinyl leukotrienes and their receptors: bridging inflammation and colorectal cancer. World J Gastroenterol. 2014;20(4):968-977. doi:10.3748/wjg.v20. i4.968

46. Kai M, Yamamoto E, Sato A, et al. Epigenetic silencing of diacylglycerol kinase gamma in colorectal cancer. Mol Carcinog. 2017;56 (7):1743-1752. doi:10.1002/mc.22631

47. Kandell RL, Bernstein C. Bile salt/acid induction of DNA damage in bacterial and mammalian cells: implications for colon cancer. Nutr Cancer. 1991;16(3-4):227-238. doi:10.1080/01635589109514161

48. Reddy BS, Watanabe K, Weisburger JH, Wynder EL. Promoting effect of bile acids in colon carcinogenesis in germ-free and conventional F344 rats. Cancer Res. 1977;37(9):3238-3242.

49. Ocvirk S, O'Keefe SJ. Influence of bile acids on colorectal cancer risk: potential mechanisms mediated by diet - gut microbiota interactions. Curr Nutr Rep. 2017;6(4):315-322. doi:10.1007/ s13668-017-0219-5

50. McGarr SE, Ridlon JM, Hylemon PB. Diet, anaerobic bacterial metabolism, and colon cancer: a review of the literature. J Clin Gastroenterol. 2005;39(2):98-109.

51. Gonzalez A, Varo N, Alegre E, Diaz A, Melero I. Immunosuppression routed via the kynurenine pathway: a biochemical and pathophysiologic approach. Adv Clin Chem. 2008;45:155-197.

52. Prendergast GC, Smith C, Thomas S, et al. Indoleamine 2,3-dioxygenase pathways of pathogenic inflammation and immune escape in cancer. Cancer Immunol Immunother. 2014;63(7):721-735. doi:10.1007/s00262-014-1549-4

53. Zhang $\mathrm{R}, \mathrm{Li} \mathrm{H}, \mathrm{Yu} \mathrm{J}$, et al. Immunoactivative role of indoleamine 2,3-dioxygenase in gastric cancer cells in vitro. Mol Med Rep. 2011;4 (1):169-173. doi: $10.3892 / \mathrm{mmr} .2010 .398$ 
54. Davis VW, Bathe OF, Schiller DE, Slupsky CM, Sawyer MB. Metabolomics and surgical oncology: potential role for small molecule biomarkers. J Surg Oncol. 2011;103(5):451-459. doi:10.1002/ jso. 21831

55. Ivanisevic J, Zhu ZJ, Plate L, et al. Toward 'omic scale metabolite profiling: a dual separation-mass spectrometry approach for coverage of lipid and central carbon metabolism. Anal Chem. 2013;85 (14):6876-6884. doi:10.1021/ac401140h
56. Buscher JM, Czernik D, Ewald JC, Sauer U, Zamboni N. Crossplatform comparison of methods for quantitative metabolomics of primary metabolism. Anal Chem. 2009;81(6):2135-2143. doi: $10.1021 / \mathrm{ac} 8022857$

\section{Publish your work in this journal}

OncoTargets and Therapy is an international, peer-reviewed, open access journal focusing on the pathological basis of all cancers, potential targets for therapy and treatment protocols employed to improve the management of cancer patients. The journal also focuses on the impact of management programs and new therapeutic

Submit your manuscript here: https:/www.dovepress.com/oncotargets-and-therapy-journal agents and protocols on patient perspectives such as quality of life, adherence and satisfaction. The manuscript management system is completely online and includes a very quick and fair peer-review system, which is all easy to use. Visit http://www.dovepress.com/ testimonials.php to read real quotes from published authors. 